\title{
Modeling and Performance Analysis of WDM Transmission Links Employing Semiconductor Optical Amplifiers
}

\author{
Jean Jennen, Huug de Waardt, and Gerard Acket
}

\begin{abstract}
We theoretically explored the system limits of various single- and multichannel transmission links employing semiconductor optical amplifiers (SOAs). For this purpose, we developed an accurate model to describe the amplifier dynamics in the moderate and low saturation regimes. Our simulation results revealed the delicate balance among the level of saturation, the signal-tonoise ratio, and the amount of gain per amplifier required for optimum system performance. The modeling results have been confirmed by recent experimental work in this area.
\end{abstract}

Index Terms-Optical communication, semiconductor optical amplifiers (SOAs), wavelength division multiplexing (WDM).

\section{INTRODUCTION}

D UE TO their fast carrier dynamics, semiconductor optical amplifiers (SOAs) have been generally considered less suited for linear amplification compared to erbium-doped fiber amplifiers (EDFAs). On the other hand, the phenomenon makes them very interesting for nonlinear applications [1].

Careful adjustment of operation conditions, however, renders SOAs into capable devices for inline amplification of both single- and multichannel systems. Recently, the SOA has generated more and more interest as a functional device in the area of long-haul optical transmission demonstrated by the results reported in [2]-[4]. We performed various simulations of single-channel and wavelength division multiplexing (WDM) systems to assess the system performance to be expected of such transmission systems when employing SOAs. As our study focused on amplifier performance, neither dispersion nor other fiber nonlinearities were included for simplicity.

Although we concentrated on application of 1310-nm SOAs, our simulation results basically are also applicable to transmission systems that are operated outside the 1310-nm window. This is important in view of Lucent Technologies' AllWave fiber. The elimination of the waterpeak around $1400 \mathrm{~nm}$ opened up the entire wavelength region from 1300 to $1600 \mathrm{~nm}$. As opposed to EDFAs, SOAs potentially cover this entire window easily.

Before giving a detailed description of the link simulations and corresponding results, we first will examine the applied SOA model. We address the rate equations on which the

Manuscript received October 17, 2000; revised April 23, 2001. This work was supported by the Dutch Technology Foundation STW.

J. Jennen is with the Forward Looking Work Department, Bell Labs, Lucent Technologies Nederland BV, 12700 AA Huizen, The Netherlands (e-mail: jeanjennen@lucent.com).

H. de Waardt and G. Acket are with the Faculty of Electrical Engineering, Eindhoven University of Technology, 5600 MB Eindhoven, The Netherlands (e-mail: h.d.waardt@ele.tue.nl).

Publisher Item Identifier S 0733-8724(01)06260-0. model is based, their modifications and simplifications, and the model's extensiveness. We also validate the SOA model by comparing the model results to experimentally obtained data. Regarding the agreement between the experimental data and the simulation results, we were encouraged to apply our SOA model in link simulations. Moreover, we can already draw some tentative conclusions based on the outcome of the simulations presented here.

\section{BACKGROUND ON THE SOA MODEL}

As opposed to the models found in [5]-[8], ${ }^{1}$ where physical material parameters and the drive current are used as inputs, we restrict ourselves to describe the SOA behavior in a phenomenological manner. Our objective is to find operational settings of the amplifiers in terms of gain, saturation behavior, and noise figure for specific applications that can easily be retrieved by measurements.

The functionality of our model becomes clear when looking at which quantities are taken into account and what matters are left out. Included in our SOA model are

1) a time-resolved description of the amplification process;

2) the wavelength dependence of the amplifier gain;

3) the wavelength dependence of the amplifier saturation behavior;

4) the amplifier polarization dependence.

To determine the influence of an SOA on the performance of (digital) transmission systems, we need to understand its transient behavior. This requires a time-resolved amplifier model. When observing WDM systems, we should include the wavelength dependence of the essential amplifier parameters. We assume the unsaturated fiber-to-fiber gain as a function of the wavelength to be Gaussian [10, ch. 5]. Thus, expressing the amplifier gain in decibels, we may write

$$
G=-12\left(\frac{\lambda-\lambda_{p}}{\Delta \lambda}\right)^{2}+G_{p}
$$

where $G_{p}$ is the peak gain at corresponding wavelength $\lambda_{p}$. The factor 12 is determined by the definition of $\Delta \lambda$, representing the full-width at half-maximum (FWHM) gain bandwidth. The 3-dB saturation output power as a function of $\lambda$ is described by the linear relationship [11], [10]

$$
P_{\mathrm{sat} 3 \mathrm{~dB}}=\wp_{\mathrm{sat}}\left(\lambda-\lambda_{p}\right)+P_{\mathrm{sat}}
$$

${ }^{1}$ See also the erratum to [6] presented in [9]. 
where $P_{\text {sat }_{\mathrm{p}}}$ equals the $3-\mathrm{dB}$ saturation output power at peak gain wavelength $\lambda_{p}$ and $\wp_{\text {sat }}$ is the linear coefficient. Inclusion of the SOA polarization dependence does not complicate the model and offers the possibility of simulating systems containing polarization-dependent amplifiers. This option, however, was not used in the simulations presented here. The commercial availability of SOAs showing residual polarization sensitivities below $0.5 \mathrm{~dB}$ justifies the ignorance of this parameter.

Not included in our model are

1) the dependence of the carrier lifetime $\tau_{c}$ on the carrier density;

2) a time-space resolved description of the amplification process;

3) the polarization dependence of the input and output coupling efficiencies $\eta_{\text {in }}$ and $\eta_{\text {out }}$, the internal cavity loss $\alpha$, and the linewidth enhancement factor $\alpha_{h}$;

4) the wavelength dependence of the spontaneous emission factor $n_{\mathrm{sp}}$, the input and output coupling efficiencies, the internal cavity loss, and the linewidth enhancement factor;

5) the residual facet reflectivities.

The carrier lifetime depends on the carrier density as follows:

$$
\frac{1}{\tau_{c}}=A_{n r}+B N+C N^{2}
$$

However, including (3) complicates our model considerably, and knowledge about the amplifier materials is required to obtain the values for $A_{n r}, B$, and $C$. Because a small change in carrier density already corresponds to a considerable change in gain, we assume $\tau_{c}$ to be constant.

Below, it is shown that we can eliminate the details of the spatial dependence of the gain, as we are only interested in the amplifier output signal (at $z=L$ ) as a function of the optical input signal (at $z=0$ ).

The input and output coupling efficiencies, the internal cavity loss, and the linewidth enhancement factor are extracted from measurements and show no substantial polarization and wavelength dependence. The wavelength independence of the spontaneous emission factor is left out for simplicity, but not for fundamental reasons. However, we expect this to introduce only a small error.

Present-day generations of traveling-wave SOAs exhibit residual facet reflectivities well below $1 \times 10^{-5}$. Gain ripple values are therefore reduced to $0.3 \mathrm{~dB}$ or less. Thus, leaving the residual facet reflectivities out is also permissible.

Our model uses as a starting point the following gain rate equation [10]:

$$
\begin{aligned}
\frac{\partial g(\psi, \nu)}{\partial t} & \frac{g_{0}(\psi, \nu)-g(\psi, \nu)}{\tau_{c}}+ \\
& \frac{\nu \sum_{i} \frac{g\left(s, \nu_{i}\right) P\left(s, \nu_{i}\right)+g\left(p, \nu_{i}\right) P\left(p, \nu_{i}\right)}{\nu_{i}}}{E_{\mathrm{sat}}(\psi, \nu)}
\end{aligned}
$$

Here, $g$ and $g_{0}$ are the actual and small-signal gains of the amplifier, respectively, and $E_{\text {sat }}$ is the saturation energy, i.e., saturation power $\times$ carrier lifetime, at the observed polarization $\psi$ and optical frequency $\nu$. The parameter $\psi$ equals $s$ or $p$ designating transverse magnetic (TM) or transverse electric (TE) polarization mode, respectively. $P$ represents the power of the optical field. Equation (4) takes the carrier depletion by multiple wavelengths into account, because an electron-hole pair that is recombined by an arbitrary wavelength is lost for all other wavelengths as well. The total number of photons determines the amount of gain depletion over the entire frequency spectrum.

The evolution of the envelope $A$ of the optical field is governed by [12]

$$
\frac{\partial A}{\partial z}+\frac{1}{v_{g}} \frac{\partial A}{\partial t}=\frac{1}{2}\left(1-i \alpha_{h}\right) g A-\frac{1}{2} \alpha A
$$

where $v_{g}$ denotes the group velocity, $\alpha_{h}$ is the linewidth enhancement factor, and $\alpha$ represents the internal cavity loss. For simplicity, the optical confinement factor of the field is left out, as it will indirectly be included via the measured small-signal gain. Because we are only interested in the envelope of the electrical field traveling with the light pulse, we introduce the following substitution:

$$
\tau=t-\frac{z}{v_{g}}
$$

Transformation of a variable $f(t, z)$ to $f(\tau, z)$ leads to the following expressions for the time and spatial derivatives of variable $f$ :

$$
\begin{aligned}
\frac{\partial f(t, z)}{\partial t} & =\frac{\partial f(\tau, z)}{\partial \tau} \cdot \frac{\partial \tau}{\partial t}=\frac{\partial f(\tau, z)}{\partial \tau} \\
\frac{\partial f(t, z)}{\partial z} & =\frac{\partial f(\tau, z)}{\partial z}+\frac{\partial f(\tau, z)}{\partial \tau} \cdot \frac{\partial \tau}{\partial z} \\
& =\frac{\partial f(\tau, z)}{\partial z}-\frac{1}{v_{g}} \cdot \frac{\partial f(\tau, z)}{\partial \tau}
\end{aligned}
$$

Applying (6) to (8) and defining

$$
A=\sqrt{\text { Const } \cdot P} e^{i \varphi} \Rightarrow P=|A|^{2} / \text { Const }
$$

we arrive at

$$
\begin{aligned}
& \frac{\partial P}{\partial z}=(g-\alpha) P \\
& \frac{\partial \varphi}{\partial z}=-\frac{1}{2} \alpha_{h} g
\end{aligned}
$$

and

$$
\begin{aligned}
\frac{\partial g(\psi, \nu)}{\partial \tau}= & \frac{g_{0}(\psi, \nu)-g(\psi, \nu)}{\tau_{c}}+ \\
& -\frac{\nu \sum_{i} \frac{g\left(s, \nu_{i}\right) P\left(s, \nu_{i}\right)+g\left(p, \nu_{i}\right) P\left(p, \nu_{i}\right)}{\nu_{i}}}{E_{\mathrm{sat}}(\psi, \nu)}
\end{aligned}
$$


When the input signal at $z=0$ is given, we can derive the output signal at $z=L$ by integrating (10) and (11) over $z=0$ to $z=L$. This results in

$$
\begin{aligned}
& P_{\text {out }}(\tau)=P_{\text {in }}(\tau) e^{g_{L}(\tau)-\alpha L} \\
& \varphi_{\text {out }}(\tau)=\varphi_{\text {in }}(\tau)-\frac{1}{2} \alpha_{h} g_{L}(\tau)
\end{aligned}
$$

where

$$
g_{L}(\tau)=\int_{0}^{L} g(z, \tau) d z
$$

We need to find a differential equation for $g_{L}(\tau)$. Therefore, we integrate (12) from $z=0$ to $z=L$

$$
\begin{aligned}
& \frac{\partial g_{L}(\psi, \nu)}{\partial \tau} \\
& =\frac{g_{0}(\psi, \nu) L-g_{L}(\psi, \nu)}{\tau_{c}}+ \\
& \quad-\frac{\int_{0}^{L} \nu \sum_{i} \frac{g\left(s, \nu_{i}\right) P\left(s, \nu_{i}\right)+g\left(p, \nu_{i}\right) P\left(p, \nu_{i}\right)}{\nu_{i}} d z}{E_{\mathrm{sat}}(\psi, \nu)} .
\end{aligned}
$$

The powers $P\left(s, \nu_{i}\right)$ and $P\left(p, \nu_{i}\right)$, respectively, represent the TM and TE contributions of all amplified external signals and the amplified spontaneous emission (ASE) at position $z$. Using $\psi$ to indicate the state of polarization, these powers can be written as

$$
\begin{aligned}
P\left(\psi, \nu_{i}, z\right)= & P_{\operatorname{in}}\left(\psi, \nu_{i}, \tau\right) \exp \left(\int_{0}^{z}\left[g\left(\psi, \nu_{i}, z^{\prime}\right)-\alpha\right] d z^{\prime}\right) \\
& +h \nu_{i} \Delta \nu n_{s p}(\psi) \int_{0}^{z} g\left(\psi, \nu_{i}, z^{\prime}\right) \\
& \cdot \exp \left(\int_{z^{\prime}}^{z}\left[g\left(\psi, \nu_{i}, z^{\prime \prime}\right)-\alpha\right] d z^{\prime \prime}\right) d z^{\prime} .
\end{aligned}
$$

Here, $\Delta \nu$ stands for the resolution bandwidth that is used for the discretization of the ASE spectrum. The first term of (17) represents the (amplified) contributions of the optical input signals, and the rightmost term takes the ASE emission into account.

Substituting (17) into (16) and solving the integral of the rightmost term in (16), we obtain the desired differential equation for $g_{L}$.

\section{VALIDATION OF THE SOA MODEL}

Before applying our SOA model in simulations of transmission systems, we first verified the amplifier dc behavior. The model should at least reproduce the SOA properties at the conditions used as model inputs, i.e., the unsaturated gain condition and the $3-\mathrm{dB}$ saturation state. We verified the dc behavior by measuring the gain-versus-output signal power curves and comparing them to the corresponding simulation results for different SOA operating conditions and signal wavelengths.

Fig. 1 shows the simulation results of a quantum-well laser amplifier (QWLA) for an input signal wavelength equal to $1310 \mathrm{~nm}$. The solid curves were generated with our model, and the symbols represent the experimental data. Fig. 1 is

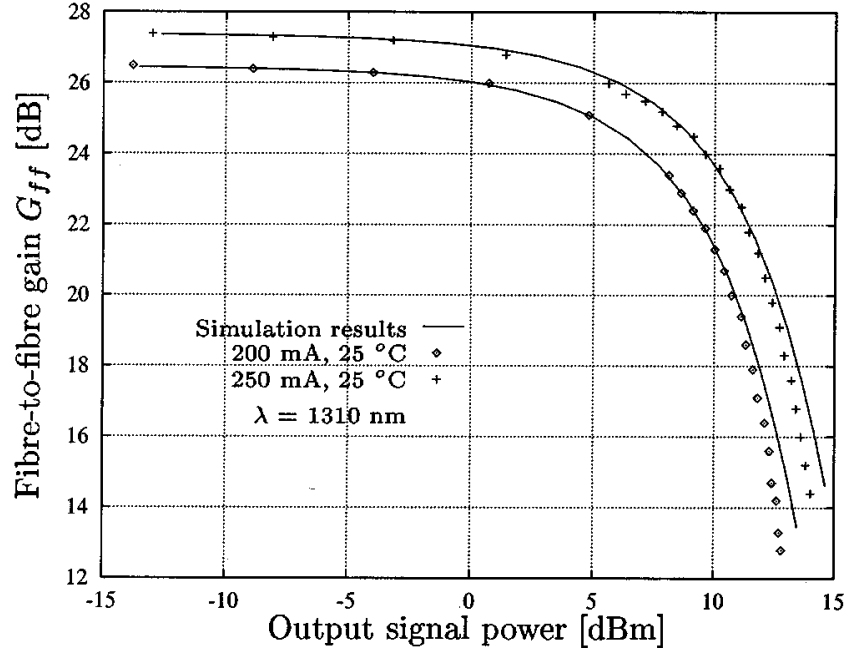

Fig. 1. Simulation results (solid curves) compared to the experimental data of a QWLA for drive currents of $200 \mathrm{~mA}(\diamond)$ and $250 \mathrm{~mA}(+)$.

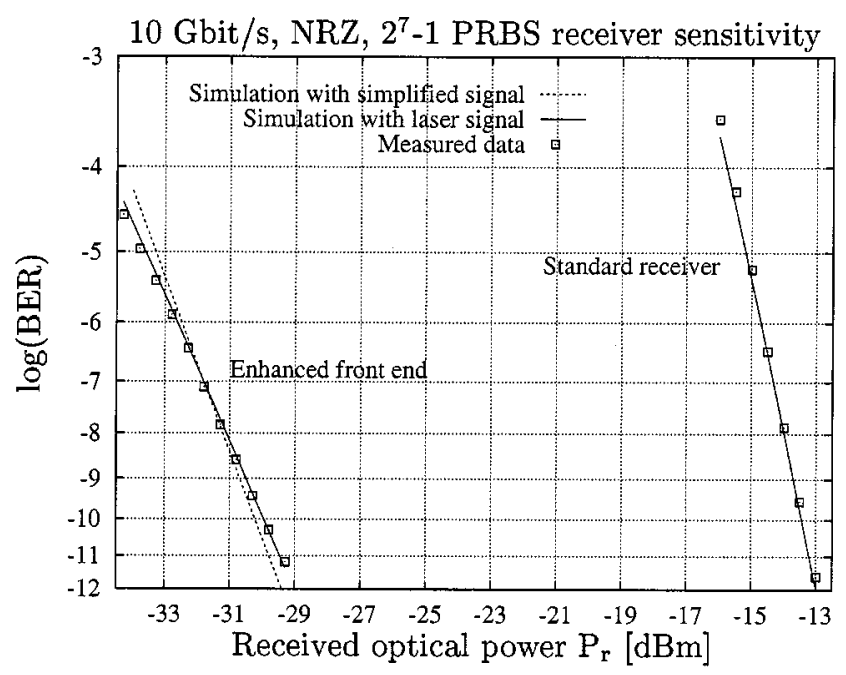

Fig. 2. Measured and simulated BER curves for both standard and enhanced front-end receivers. The simulation curves of the standard receiver coincide. Here, $I_{\mathrm{SOA}}=200 \mathrm{~mA}$ in the case of the EFE receiver.

illustrative for all comparisons made between our simulation results and the experimental data of other SOAs.

From Fig. 1 and the other results, we conclude that our model describes the static behavior of SOAs very well as long as saturation is limited to about $9 \mathrm{~dB}$. At a gain saturation level of $13 \mathrm{~dB}$, the maximum observed difference in corresponding output signal powers between the simulations and the measurements equals $1 \mathrm{~dB}$. Practical saturation levels remain within $3 \mathrm{~dB}$; otherwise, transmission capacity would be reduced considerably. So, in a vast majority of cases, our model is applicable.

An even more convincing example of the model's capabilities is demonstrated in [13], where we compared the experimental results of an optically preamplified standard PIN receiver (enhanced front-end receiver) to the corresponding simulation results. In Fig. 2, the resulting bit error rate (BER) curves from both experiments and simulations are depicted for an SOA drive current of $200 \mathrm{~mA}$. For other drive currents, comparable results 


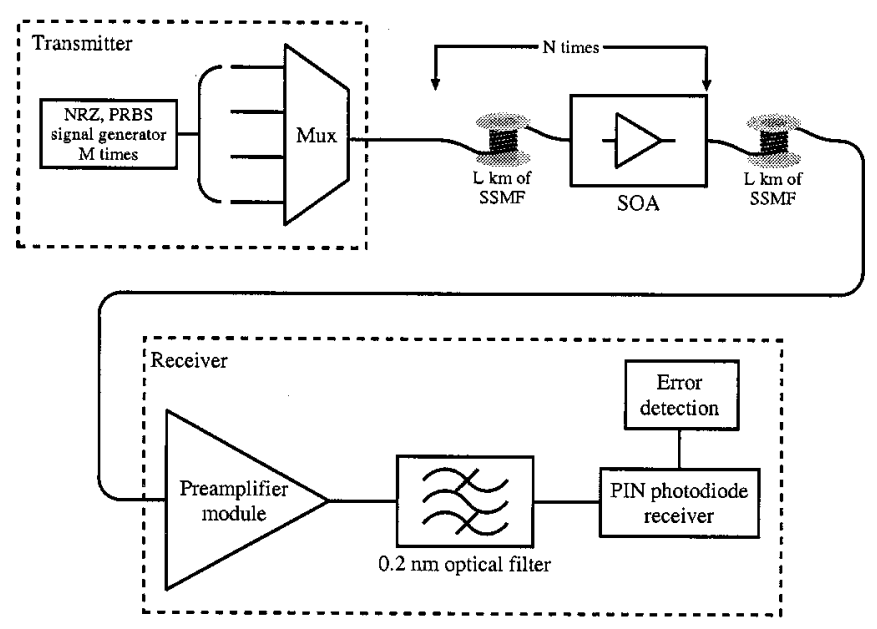

Fig. 3. Simulation model for long-haul transmission links.

were obtained. To arrive at the BER values in our simulations, we applied the noise equations given by [14].

The simulation results show good agreement with the experimental data. Fig. 2 indicates that the best match with the experiments is achieved by simulations based on a realistic laser signal, i.e., inclusion of relaxation oscillations. The maximum difference between the experimental data and the simulation results amounts to 0.15 (laser signal) and $0.5 \mathrm{~dB}$ (simplified signal). Both values are acceptable for a single amplifier stage. However, when simulating amplifier cascades, we should choose the appropriate optical signal in order to restrict the cumulative error.

\section{Description of the Simulation Runs}

As a representative simulation model for long-haul transmission links, we used the one depicted in Fig. 3. The transmitter was modeled as $M$ independent nonreturn-to-zero (NRZ) signal generators that were multiplexed before launching into the link. We adopted a $2^{7}-1$ pseudorandom bit sequence modulation format to limit the simulation time and memory requirements. At the transmitter output, the bit periods of each WDM channel were synchronized. As such, the randomness of the bit position is not taken into account to avoid an excessive increase in required simulation runs. This might have some influence at situations with a low channel count at low bit rates. However, it averages out as the channel number and the bit rate increase. The average optical power per signal was kept at $2 \mathrm{dBm}$ for all simulation runs. The optical channel spacing was set to $100 \mathrm{GHz}$ ( $0.57 \mathrm{~nm}$ in the $1310-\mathrm{nm}$ window) complying with ITU standards. The center wavelength of the signal spectrum equaled $1310 \mathrm{~nm}$.

The link represented a cascade of $N+1$ fiber sections of $L \mathrm{~km}$ length connected to each other with $N$ identical inline amplifiers. These amplifiers all exhibited a peak gain wavelength of $1310 \mathrm{~nm}$, an optical bandwidth of $60 \mathrm{~nm}$, and a linewidth enhancement factor of five. In our simulations, we used optical isolators at the amplifier inputs and outputs. So, backward-traveling ASE was not an issue.

The link was terminated with a preamplified optical front end. This front end was modeled as a standard PIN photo-
TABLE I

Key PARAMETERS AND theIR RANGES AS USED IN THE SimUlations

\begin{tabular}{c|c|c} 
Parameter & Symbol & Range \\
\hline Section length & $L$ & $25-62.5 \mathrm{~km}$ \\
Excess gain per section & $G_{e}$ & $-5-+5 \mathrm{~dB}$ \\
SOA Peak gain wavelength & $\lambda_{p}$ & $1310 \mathrm{~nm}$ \\
SOA Optical bandwidth & $B W$ & $60 \mathrm{~nm}$ \\
SOA Noise figure & $N F$ & $8 \mathrm{~dB}$ \\
SOA Saturation power & $P_{s a t 3 d B}$ & $12-15 \mathrm{dBm}$ \\
Number of channels & $M$ & $1-32$ \\
Bitrate & $r_{b}$ & $2.5-20 \mathrm{Gbit} / \mathrm{s}$
\end{tabular}

diode receiver with the decision threshold optimized for the incoming signal. In front of the receiver we applied a $0.2-\mathrm{nm}$ optical bandpass filter. The electrical bandwidth was set to 0.75 times the bit rate.

Before discussing the simulations, we observe the parameter space. Because we had to deal with a considerable number of parameters, we carefully considered what type of information is valuable to system designers. Basically, the starting point for a system designer is the given span length between the amplifiers or repeaters. Reduction of regenerators in optical transmission networks is essential to limit the system costs and enhance flexibility. So, for a given section length, it is preferred to determine the maximum attainable link length and the corresponding amplifier requirements when transmitting a certain number of wavelength channels at a particular bit rate. On the other hand, when the distance between the regenerators is fixed, one wants to find out what transmission capacity can be reached depending on the choice of the inline amplifiers.

The cascadability of SOAs has already been studied in the past: results can be found in [14]-[16]. Here, we intend to increase the understanding of SOA cascades in optical high-speed transmission systems by looking at a broader parameter space than observed before.

To get a feeling for the issues presented above, we performed the simulations while observing the parameter settings for the inline amplifiers, as depicted in Table I. This table also indicates the range considered for each parameter. Here, we define the excess gain per section $G_{e}$ as the unsaturated gain in decibels of each inline amplifier minus the equivalent section loss in decibels $(0.4 \mathrm{~dB} / \mathrm{km} \times L)$. So, in the absence of saturation, a positive excess gain results in an increasing signal power along the link, and vice versa for a negative excess gain. For the preamplifier, we maintained a gain of $30 \mathrm{~dB}$, a noise figure of $8 \mathrm{~dB}$, and a saturation output power of $12 \mathrm{dBm}$ in all simulation runs.

In our simulations, we treated the SOA gain and the SOA saturation power as independent variables. This approach is justified because, in practice, these parameters can be changed virtually independently by temperature tuning in addition to bias current adjustment [15], [17].

\section{DETERMinATION OF System Limits}

Per combination of bit rate $\left(r_{b}\right)$, noise figure $(N F)$, saturation power $\left(P_{\mathrm{sat} 3 \mathrm{~dB}}\right)$, and section length $(L)$, a three-dimensional (3-D) surface plot was generated. In Figs. 4 and 5, some illustrative examples are given. The 3-D plots show the maximum 


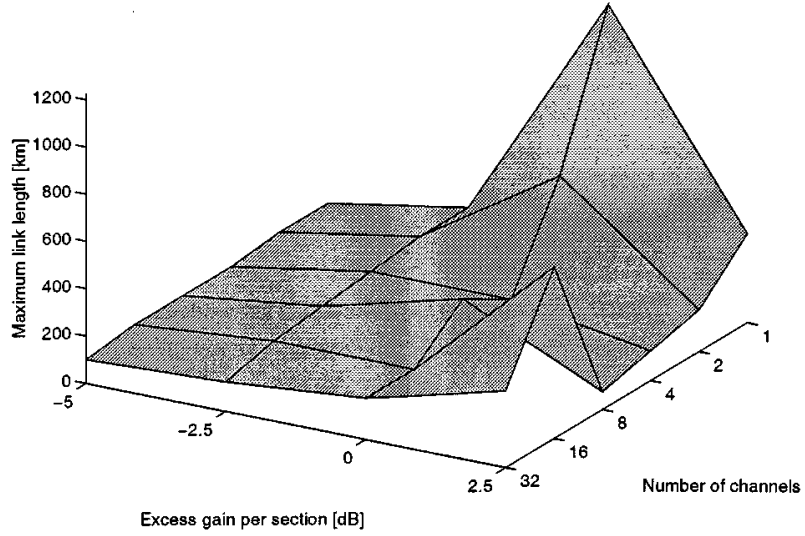

Fig. 4. 3-D surface plot of the maximum attainable link length in kilometers as a function of the number of channels and the excess gain per section for $r_{b}=$ $2.5 \mathrm{~Gb} / \mathrm{s}, \lambda_{p}=1310 \mathrm{~nm}, B W=60 \mathrm{~nm}, N F=8 \mathrm{~dB}, P_{\text {sat } 3 \mathrm{~dB}}=12 \mathrm{dBm}$, and $L=25 \mathrm{~km} \equiv 10 \mathrm{~dB}$. The surface represents an isober of $1 \times 10^{-12}$.

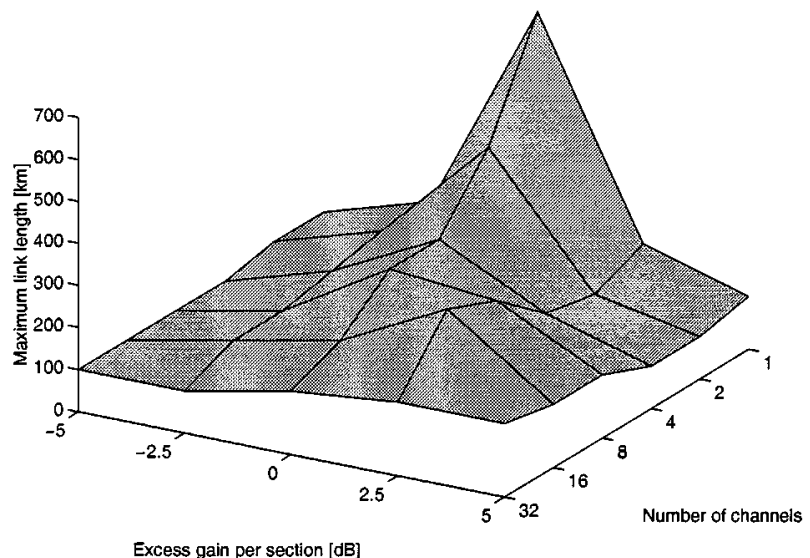

Fig. 5. 3-D surface plot of the maximum attainable link length in kilometers as a function of the number of channels and the excess gain per section for $r_{b}=10 \mathrm{~Gb} / \mathrm{s}, \lambda_{p}=1310 \mathrm{~nm}, B W=60 \mathrm{~nm}, N F=8 \mathrm{~dB}, P_{\mathrm{sat} 3 \mathrm{~dB}}=12 \mathrm{dBm}$, and $L=25 \mathrm{~km} \equiv 10 \mathrm{~dB}$. The surface represents an isober of $1 \times 10^{-12}$.

attainable distances as a function of both the number of channels and the excess gain per section for different parameter sets. In all cases, we took a minimum required BER of $1 \times 10^{-12}$ for the worst performing channel as the criterion for the maximum attainable distance. Therefore, each 3-D surface may be considered as an isober at $1 \times 10^{-12}$. The volumes below the surfaces correspond to a higher performance.

Figs. 4 and 5 show the general trend of reduced BER performance with increasing distance and/or less gain per amplifier section. In both cases, a reduced signal-to-noise ratio (SNR) hampers system performance. This fully agrees with our expectations. In the figures, we also observe two other trends that require some reflection.

1) With increasing gain per amplifier section, the system performance first increases, reaches a maximum, and keeps decreasing thereafter.

2) Along the Number of channels axis, the system performance does not show a continuously descending level under all circumstances.

The first observation is not that surprising when considering the inevitable effect of gain saturation. In absence of saturation,
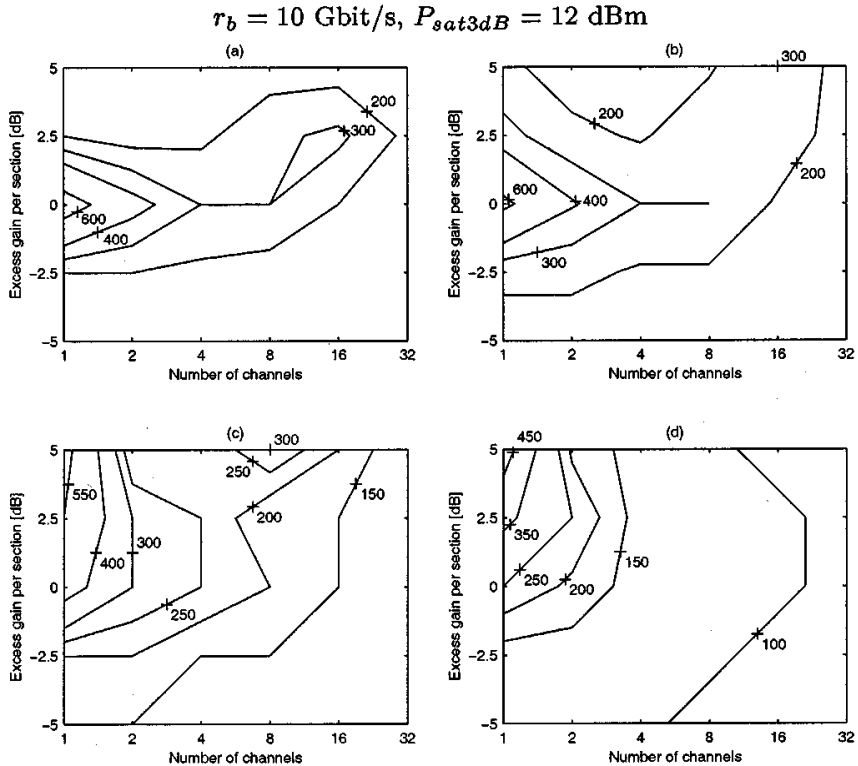

Fig. 6. Contour plot of the maximum attainable link length in kilometers as a function of the number of channels and the excess gain per section. The criterion was a minimum BER of $1 \times 10^{-12}$. Four different section lengths were chosen: (a) $L=25 \mathrm{~km} \equiv 10 \mathrm{~dB}$, (b) $L=37.5 \mathrm{~km} \equiv 15 \mathrm{~dB}$, (c) $L=50 \mathrm{~km} \equiv 20 \mathrm{~dB}$, and (d) $L=62.5 \mathrm{~km} \equiv 25 \mathrm{~dB}$.

improved system performance would undoubtedly be obtained for every raise in gain level. For single-channel transmission, the occurrence of pattern effects at high gain levels limits the system performance [18]. In WDM transmission systems, cross-gain modulation additionally contributes to the performance degradation.

To study the second observation, we use a more convenient visualization of the simulation results, i.e., two-dimensional contour plots of the corresponding 3-D surface plots (see Figs. 6-9). The angularity of the contours is due to the limited number of probed excess gains and channel counts. For the $2.5-\mathrm{Gb} / \mathrm{s} \mathrm{simu-}$ lations, a maximum excess gain of $2.5 \mathrm{~dB}$ was used; at an excess gain of $5 \mathrm{~dB}$, we encountered memory allocation problems.

When saturation effects are predominant over noise, we observe that the optimum performance is found at larger excess gains for an increasing number of channels. Figs. 6(a) and (b), 7(a) and (b), 8(a) and (b), and 9(a) and (b) clearly show this trend. Based on our observation, we conclude that somehow the saturation effects, e.g., the cross-gain modulation (XGM), are reduced when the number of channels increases. By taking a closer look at the statistical behavior of the transmitted signals, we find an explanation for that.

When a sufficiently large number of data signals is transmitted over the link, an averaging effect on the instantaneous total power occurs. This averaging effect leads to a reduction of the XGM and consequently counteracts the amount of crosstalk. Fig. 10 clearly illustrates the positive effect that results from a large number of channels. In [4], the same effect is demonstrated in a system experiment. Fig. 10 also shows some signal deterioration when going from single- to two-channel transmission. In a two-channel WDM system, the averaging effect is yet too small to compensate for the introduced crosstalk. This also explains why the maximum attainable link length reaches a minimum at two or four channels. 

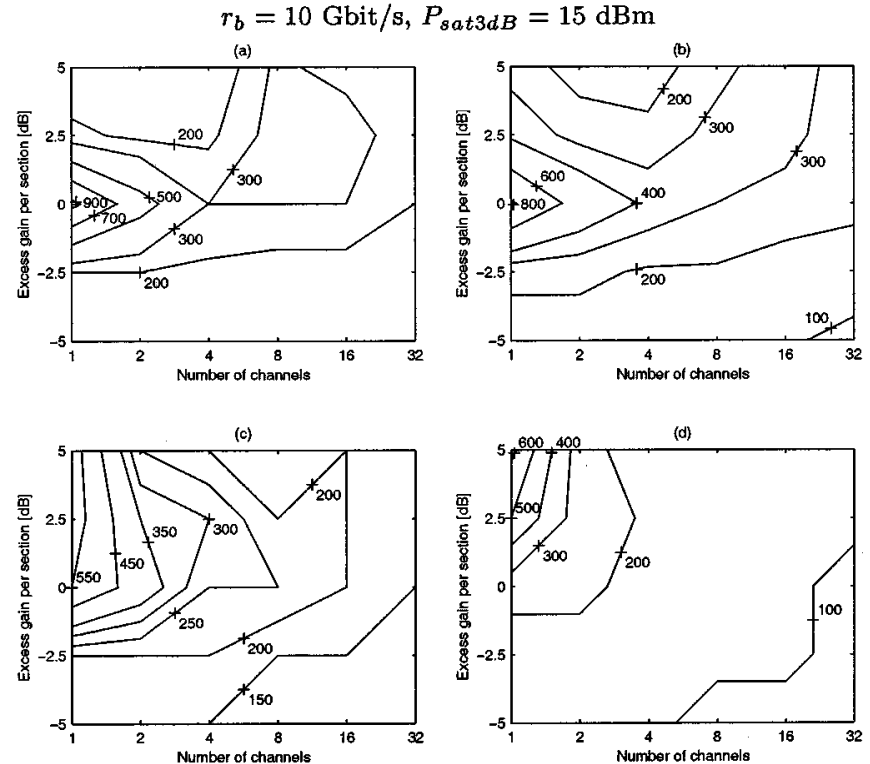

Fig. 7. Contour plot of the maximum attainable link length in kilometers as a function of the number of channels and the excess gain per section. The criterion was a minimum BER of $1 \times 10^{-12}$. Four different section lengths were chosen: (a) $L=25 \mathrm{~km} \equiv 10 \mathrm{~dB}$, (b) $L=37.5 \mathrm{~km} \equiv 15 \mathrm{~dB}$, (c) $L=50 \mathrm{~km} \equiv 20 \mathrm{~dB}$, and (d) $L=62.5 \mathrm{~km} \equiv 25 \mathrm{~dB}$.
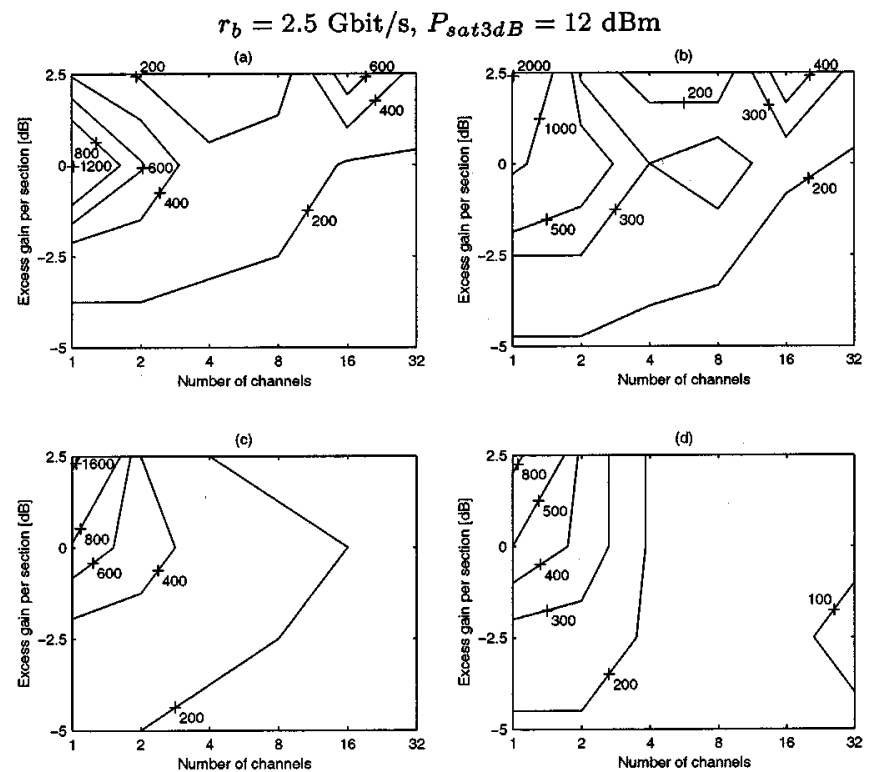

Fig. 8. Contour plot of the maximum attainable link length in kilometers as a function of the number of channels and the excess gain per section. The criterion was a minimum BER of $1 \times 10^{-12}$. Four different section lengths were chosen: (a) $L=25 \mathrm{~km} \equiv 10 \mathrm{~dB}$, (b) $L=37.5 \mathrm{~km} \equiv 15 \mathrm{~dB}$, (c) $L=50 \mathrm{~km} \equiv 20 \mathrm{~dB}$, and (d) $L=62.5 \mathrm{~km} \equiv 25 \mathrm{~dB}$.

It remains to be explained why, with a further increasing number of channels, the maximum attainable link length shows a local maximum at eight or 16 channels. Two reasons can be identified to explain this.

1) Because we kept the average signal power per channel constant in our simulations, a larger averaged amount of saturation was experienced with increasing number of channels. Each channel was launched into the link having a power level of $2 \mathrm{dBm}$. So, with eight channels, this adds up to $11 \mathrm{dBm}$. Knowing that $P_{\mathrm{sat}} 3 \mathrm{~dB}$ equals $12 \mathrm{dBm}$, an
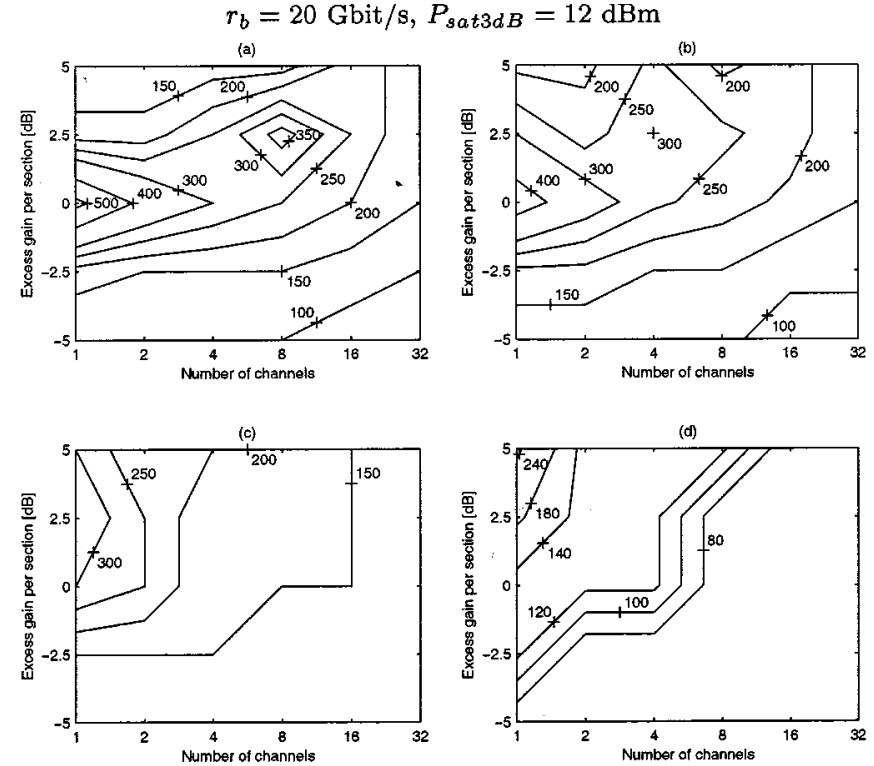

Fig. 9. Contour plot of the maximum attainable link length in kilometers as a function of the number of channels and the excess gain per section. The criterion was a minimum BER of $1 \times 10^{-12}$. Four different section lengths were chosen: (a) $L=25 \mathrm{~km} \equiv 10 \mathrm{~dB}$, (b) $L=37.5 \mathrm{~km} \equiv 15 \mathrm{~dB}$, (c) $L=50 \mathrm{~km} \equiv 20 \mathrm{~dB}$, and (d) $L=62.5 \mathrm{~km} \equiv 25 \mathrm{~dB}$.

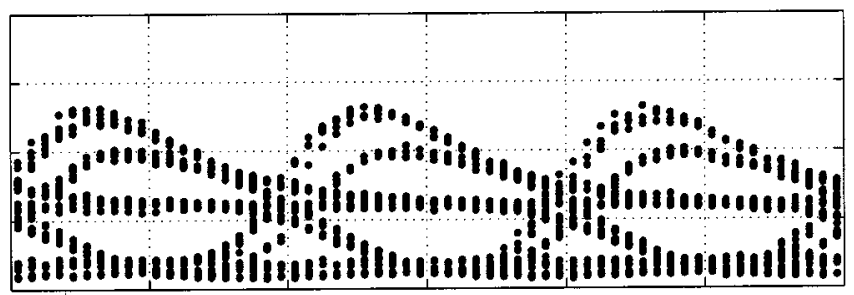

(a)

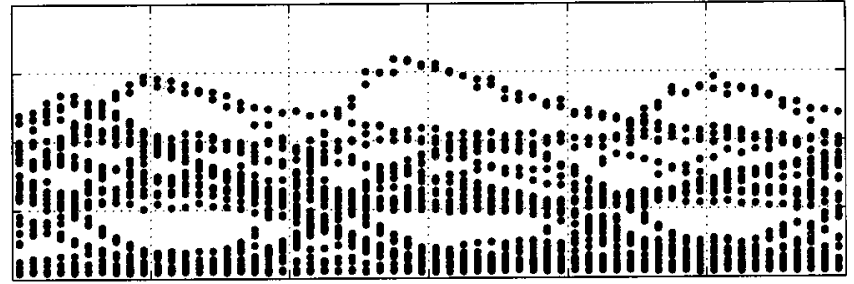

(b)

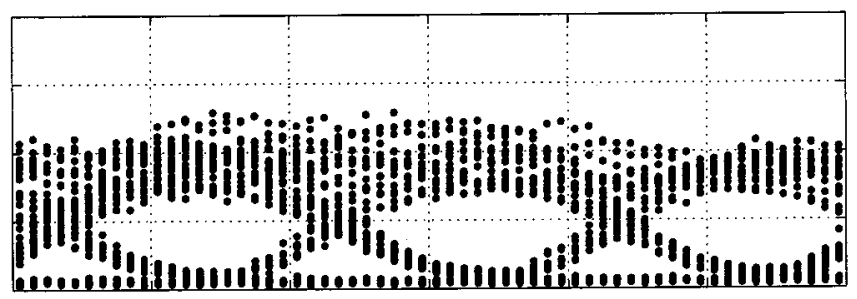

(c)

Fig. 10. Simulated eye patterns of the center wavelengths after five inline amplifiers in (a) single-channel, (b) two-channel, and (c) 32-channel transmission systems, where $r_{b}=10 \mathrm{~Gb} / \mathrm{s}, N F=8 \mathrm{~dB}, P_{\text {sat } 3 \mathrm{clB}}=12 \mathrm{dBm}$, $L=37.5 \mathrm{~km} \equiv 15 \mathrm{~dB}$, and $G_{e}=5 \mathrm{~dB}$. The horizontal resolution is $50 \mathrm{ps} / \mathrm{div}$. All three eye patterns are normalized to their respective average signal powers.

unsaturated excess gain of $2.5 \mathrm{~dB}$ will result in an experienced excess gain of about zero at such power levels.

2) Although the unsaturated amplifier bandwidth equals $60 \mathrm{~nm}$ at FWHM, more than enough to contain 32 
channels $(3.1 \mathrm{THz} \equiv 18 \mathrm{~nm})$, the effective link bandwidth is reduced by bandwidth narrowing [15] down to $27 \mathrm{~nm}$ after five amplifier stages, and $19 \mathrm{~nm}$ after ten SOAs. The optical bandwidth is even further reduced by wavelength-dependent saturation: gain saturation at the shorter wavelengths is more severe than it is at the longer wavelengths. Since we maintain the criterion that no channel should perform less than a BER of $1 \times 10^{-12}$, the shortest wavelength channel determines the maximum attainable distance.

Let us now look at the cases where the noise effects prevail over the saturation effects. When the section length $L$ is increased, we observe a shift of the optimum transmission distance from zero toward the larger excess gains for a small number of channels (1 or 2). Figs. 6(c) and (d), 7(c) and (d), 8(b)-(d), 9(d), and, to a lesser extent, Fig. 9(c) illustrate this effect. One would expect, however, that the optimum would remain at zero excess gain, as is the case for short section lengths $(25$ or $37.5 \mathrm{~km})$. For longer sections, two different matters can be distinguished that explain the shift toward positive excess gains.

1) The number of cascaded amplifiers. In the case of long sections, fewer amplifiers are cascaded and therefore larger positive excess gains per section can be allowed before reaching sizeable saturation.

2) The effect of ASE on the input power dynamic range. At equal excess gains, the gain of the amplifiers in a cascade with long sections is higher than in the case when the section lengths are short. The corresponding ASE levels are then higher as well. The input power dynamic range of the amplifiers further down the link increases due to the "clamping effect" of accumulated ASE from the previous stages.

The effect described above shows bit-rate dependence. At $2.5 \mathrm{~Gb} / \mathrm{s}$, the effect already occurs for section lengths of $37.5 \mathrm{~km}$, whereas at $20 \mathrm{~Gb} / \mathrm{s}$ the effect is less prominent for section lengths of $50 \mathrm{~km}$. This is explained by the fact that at a bit rate of $20 \mathrm{~Gb} / \mathrm{s}$, the signal suffers more from saturation than is the case at a bit rate of $2.5 \mathrm{~Gb} / \mathrm{s}$. Note that this is the case for the amplifier type with a carrier lifetime of 200 ps used in our simulations. So, for $2.5 \mathrm{~Gb} / \mathrm{s}$ (nearly) full recovery of the amplifier gain could occur within one bit time. For 10 and $20 \mathrm{~Gb} / \mathrm{s}$, gain recovery required several bit times, and consequently pattern effects occurred. Fig. 11 shows the bit-rate dependence of signal distortion induced by saturation.

To establish the influence of the saturation output power, we compare Figs. 6 and 7. As expected, the maximum attainable link length is affected the most when saturation is the dominant source of signal deterioration. This is the case for large excess gains, a small number of channels, and relatively small section lengths. Transmission distance improvements of up to $36 \%$ can be obtained by increasing the saturation output power by $3 \mathrm{~dB}$.

\section{RELATIONS TO OTHER WORK}

Comparing our results to other data found in literature, we arrive at the following findings. Our results agree with those of (a)

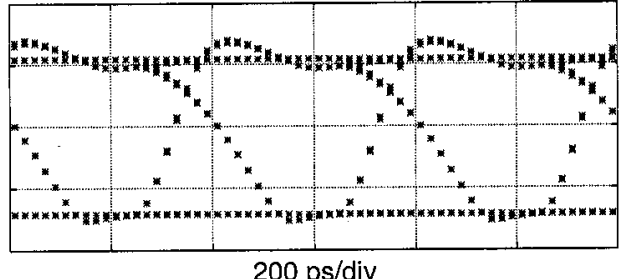

(b)

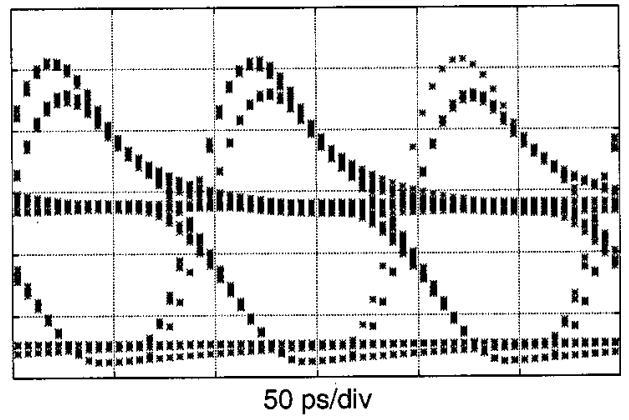

(c)

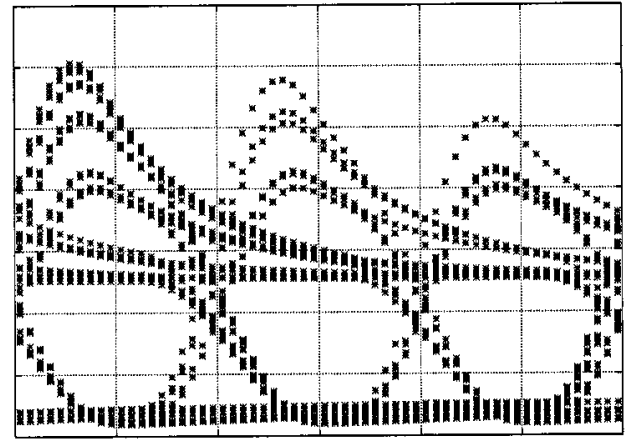

$25 \mathrm{ps} / \mathrm{div}$

Fig. 11. Simulated eye patterns after five inline amplifiers in a single channel transmission system for (a) $r_{b}=2.5 \mathrm{~Gb} / \mathrm{s}$, (b) $r_{b}=10 \mathrm{~Gb} / \mathrm{s}$, and (c) $r_{b}=20$ $\mathrm{Gb} / \mathrm{s}$. In all cases, $N F=8 \mathrm{~dB}, P_{\mathrm{sat}} 3 \mathrm{~dB}=12 \mathrm{dBm}, L=50 \mathrm{~km} \equiv 20 \mathrm{~dB}$, and $G_{e}=5 \mathrm{~dB}$. All three eye patterns are normalized to their respective average signal powers. Only the influence of saturation is shown; no noise is added.

[16]. The maximum attainable link length versus the number of channels found in Figs. 6(d) and 8(d) comply with the values derived from [16, Fig. 4]. The numbers given in [14] and [15] for the maximum amplifier cascades are not confirmed by our results. Their estimates are too optimistic because they do not take the dynamic saturation effects into account.

The comparison of the experimental results demonstrated in [4] and [19] to the outcome of our simulations is even more interesting. Let us look at Fig. 8(b) for comparison to the transmission experiment in [4]. In this experiment, gain clamping was implemented by the use of a reservoir channel. So, the inline SOAs exhibited an excess gain of about $2 \mathrm{~dB}$ (the span loss equaled $15 \mathrm{~dB}$ per section). For this excess gain and 32 channels, we find that the maximum attainable number of inline amplifiers is six. In [4], they reached a number of three inline SOAs. This can be explained by two differences between our simulations and the experiment. First, the amplifiers in the experiment had a noise figure of $10 \mathrm{~dB}$. The additional $2 \mathrm{~dB}$ reduces the theoretical limit already to four amplifiers. In addition, the average power per channel was $7.1 \mathrm{~dB}$ lower in the experiment than in 
the simulations. Thus, the operational noise figure could easily be $1 \mathrm{~dB}$ higher [10, Sec. 4.1.2].

The amplifiers used in [19] had a minimal signal gain of $12 \mathrm{~dB}$, a noise figure of $10 \mathrm{~dB}$, and a saturation output power of $12 \mathrm{dBm}$. The reported span loss equaled $13 \mathrm{~dB}$. When assigning $2 \mathrm{~dB}$ of the SOA input coupling efficiency to the span loss, the transmission link is converted to one with $15-\mathrm{dB}$ spans and inline amplifiers with noise figures of $8 \mathrm{~dB}$ and signal gains of at least $14 \mathrm{~dB}$. After doing so, we can use Fig. 9(b) for validation of the experimental result. Based on our simulation results, we find that for a transmission link as described in [19], the maximum number of inline SOAs equals four at $1 \times 10^{-12}$ BER. This fully agrees with the transmission experiment.

\section{CONCLUSION}

An SOA model was developed that shows accurate results for both single-channel and WDM transmission when moderate or low saturation levels are observed. We used this model to determine the system limits of various transmission links. We found that tradeoffs among the level of saturation, the SNR, and the amount of gain have to be made for any kind of transmission links that employ SOAs. Without any exception, single-channel transmission can bridge the largest attainable distances with respect to multichannel transmission under the same circumstances. Our simulations also demonstrate that the worst WDM system incorporating SOAs is a two-channel one. The experimental results demonstrated in [4] and [19] confirm the outcome of our simulations.

\section{ACKNOWLEDGMENT}

The authors would like to thank the reviewers for their comments, which improved this manuscript considerably.

\section{REFERENCES}

[1] K. E. Stubkjaer et al., "Wavelength conversion devices and techniques," in Proc. 22nd Eur. Conf. Optical Communication, Oslo, Norway, Sept. 1996, pp. 4.33-4.40.

[2] J. J. E. Reid et al., "An international field trial at $1.3 \mu \mathrm{m}$ using an $800 \mathrm{~km}$ cascade of semiconductor optical amplifiers," in Proc. 24th Eur. Conf. Optical Communication, vol. 1, Madrid, Spain, Sept. 1998, pp. 567-568.

[3] J. G. L. Jennen et al., " $4 \times 10 \mathrm{Gbit} / \mathrm{s}$ NRZ transmission in the $1310 \mathrm{~nm}$ window over $80 \mathrm{~km}$ of standard single mode fiber using semiconductor optical amplifiers," in Proc. 24th Eur. Conf. Optical Communication, vol. 1, Madrid, Spain, Sept. 1998, pp. 235-236.

[4] Y. Sun et al., "Error-free transmission of $32 \times 2.5 \mathrm{~Gb} / \mathrm{s}$ DWDM Channels over $125 \mathrm{~km}$ of AllWave ${ }^{\mathrm{TM}}$ fiber using cascaded in-line semiconductor optical amplifiers," in Tech. Dig. Topical Meeting on Optical Amplifiers and Applications, Nara, Japan, June 1999, pp. PdP6_1-PdP6_4.

[5] M. J. Adams et al., "Analysis of semiconductor laser optical amplifiers," Proc. Inst. Elect. Eng. Opto-Electron., vol. 132, no. 1, pp. 58-63, Feb. 1985.

[6] I. D. Henning et al., "Performance predictions from a new optical amplifier model," IEEE J. Quantum Electron., vol. QE-21, pp. 609-613, June 1985.

[7] L. Gillner et al., "Semiconductor laser amplifier optimization: An analytical and experimental study," IEEE J. Quantum Electron., vol. 25, pp. 1822-1827, Aug. 1989.

[8] P. Brosson, "Analytical model of a semiconductor optical amplifier," $J$. Lightwave Technol., vol. 12, pp. 49-54, Jan. 1994.

[9] I. D. Henning et al., "Correction to 'Performance predictions from a new optical amplifier model'," IEEE J. Quantum Electron., vol. QE-21, p. 1973, Dec. 1985.
[10] J. G. L. Jennen, "Noise and saturation effects in high-speed transmission systems with semiconductor optical amplifiers," Ph.D. dissertation, Eindhoven Univ. Technol., Eindhoven, The Netherlands, June 2000.

[11] L. F. Tiemeijer et al., "Polarization resolved, complete characterization of $1310 \mathrm{~nm}$ fiber pigtailed multiple-quantum-well optical amplifiers," $J$. Lightwave Technol., vol. 14, pp. 1524-1533, June 1996.

[12] G. P. Agrawal and N. A. Olsson, "Amplification and compression of weak picosecond optical pulses by using semiconductor-laser amplifiers," Opt. Lett., vol. 14, no. 10, pp. 500-502, May 1989.

[13] J. G. L. Jennen et al., "Record $10 \mathrm{Gbit} / \mathrm{s}$, NRZ receiver sensitivity at 1316 nm using a quantum well laser amplifier," in Proc. 1996 IEEE/LEOS Symp. Benelux Chapter, Enschede, The Netherlands, Nov. 1996, pp. 124-127.

[14] N. A. Olsson, "Lightwave systems with optical amplifiers," J. Lightwave Technol., vol. 7, pp. 1071-1082, July 1989.

[15] L. F. Tiemeijer et al., "Temperature dependence of a $1300 \mathrm{~nm}$ polarization-insensitive multiple quantum well laser amplifier and its implications for the ultimate capacity of cascaded amplifier systems," IEEE Photon. Technol. Lett., vol. 6, pp. 1300-1302, Nov. 1994.

[16] D. Wolfson and K. E. Stubkjaer, "Comparison of the cascadability of conventional and gain-clamped semiconductor optical amplifier gates in multi wavelength optical networks," in Proc. Conf. Optical Fiber Communication, San Jose, CA, Feb. 1999, pp. 45-47.

[17] L. F. Tiemeijer et al., " $27 \mathrm{~dB}$ gain unidirectional $1300 \mathrm{~nm}$ polarization-insensitive multiple quantum well laser amplifier module," IEEE Photon. Technol. Lett., vol. 6, pp. 1430-1432, Dec. 1994.

[18] K. Inoue, "Waveform distortion in a gain-saturated semiconductor optical amplifier for NRZ and Manchester formats," Proc. Inst. Elect. Eng. Opto-Electron., vol. 144, no. 6, pp. 433-437, Dec. 1997.

[19] L. H. Spiekman et al., "160 Gbit/s $(8 \times 20 \mathrm{Gbit} / \mathrm{s})$ DWDM transmission over $160 \mathrm{~km}$ of standard fiber using a cascade of semiconductor optical amplifiers," in Proc. 25th Eur. Conf. Optical Communication, Nice, France, Sept. 1999, pp. 36-37.

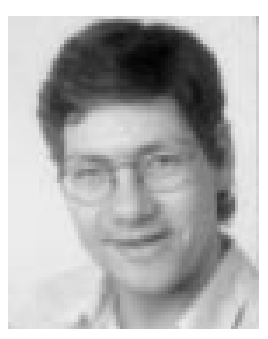

Jean Jennen was born in Beek(L), The Netherlands, on January 24, 1971. He received the M.Sc. degree (cum laude) in electrical engineering and the Ph.D. degree from the Eindhoven University of Technology, Eindhoven, The Netherlands, in 1994 and 2000 , respectively.

His doctoral research concerned the system performance of 1310-nm semiconductor optical amplifiers. In July 1998, he joined the Forward Looking Work Department of Lucent Technologies, Huizen, The Netherlands, where he is involved in the design of advanced optical communication systems.

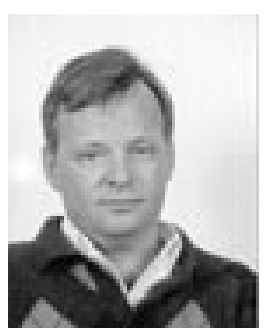

Huug de Waardt was born in Voorburg, The Netherlands, on December 1, 1953. He received the M.Sc. and Ph.D. degrees in electrical engineering from the Delft University of Technology, Delft, The Netherlands, in 1980 and 1995, respectively.

In 1981, he joined the Department of Physics, KPN Research, Leidschendam, The Netherlands, where he was engaged in research on the performance aspects of long-wavelength semiconductor laser diodes, LEDs, and photodiodes. In 1989, he joined the Department of Transmission, where he has been working in the fields of high bit rate direct-detection systems, optical preamplification, wavelength division multiplexing, dispersion-related system limitations, and the system application of resonant optical amplifiers. He contributed to international standardization bodies and to the EURO-COST activities 215 and 239. In October 1995, he became an Associate Professor at the Faculty of Electrical Engineering, University of Eindhoven, in the area of high-speed trunk transmission. His current research interests are in applications of semiconductor optical amplifiers, high-speed OTDM transmission, integrated optical cross-connects, and WDM optical networking. He was active in European research programs as ACTS BLISS and ACTS Upgrade. At present, he is responsible for the WDM system demonstrator within the ACTS APEX project. 


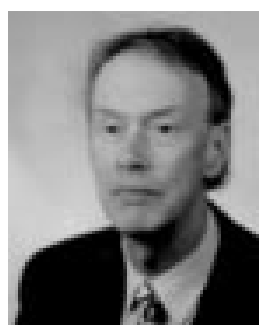

Gerard Acket was born in Utrecht, The Netherlands, in 1935. He received the Ph.D. degree in physics from Utrecht University, Utrecht, in 1965.

His doctoral work was related to the semiconducting properties of some transition metal oxides. In 1966, he joined Philips Research Laboratories, Eindhoven, The Netherlands, working on microwave instabilities and hot electron effects in III-V semiconductors. In 1972, he became involved in work on optical properties of semiconductors and semiconductor lasers. In 1976, he became a Department Head. In 1981, he became a part-time Professor at Delft University of Technology, Delft, The Netherlands, where he started work on integrated optics for optical communications, notably for WDM. In 1991, he became a part-time Professor at Eindhoven University of Technology, Eindhoven, The Netherlands, working on advanced laser structures. He retired from Philips in 1995. He published more than 50 papers and patents, including a book chapter on visible laser diodes.

Prof. Acket is a member of the Royal Dutch Academy of Sciences. 